\title{
Models of Type Ia Supernova Explosions
}

\author{
J.C. Niemeyer, M. Reinecke and W. Hillebrandt \\ Max-Planck-Institut für Astrophysik, Karl-Schwarzschild-Str. 1, 85748 Garch- \\ ing, Germany
}

\begin{abstract}
Type Ia supernovae have become an indispensable tool for studying the expansion history of the universe, yet our understanding of the explosion mechanism is still incomplete. We describe the variety of discussed scenarios, sketch the most relevant physics, and report recent advances in multidimensional simulations of Chandrasekhar mass white dwarf explosions.
\end{abstract}

\section{$0.1 \quad$ Introduction}

The comparison of the luminosity distances of low and high redshift samples of type Ia supernovae (SNe Ia) is the central pillar of the claim that our universe is accelerating [1], 2]. The observational techniques, data analysis (including the light curve shape corrections), and potential sources of systematic errors have been discussed and reviewed (e.g. [3, 仼) extensively in the literature. One, perhaps the most important, remaining uncertainty is the possible evolution of the supernova sample. Some or even all of the observed dimming of SNe Ia at $z \approx 1$ may still be the result of an intrinsic trend of peak brightness with cosmic age that is not, or only incompletely, accompanied by the corresponding change of the light curve width. Empirically, all of the observed correlations of supernova brightness with stellar population seem to disappear after the light curve shape correction, hence no such trend is evident 5. On the other hand, it is very difficult to extrapolate this result to higher redshifts without a solid theoretical understanding of the origin of these correlations and of the physics of the brightness-decline relation itself. This, in turn, requires the construction of self-consistent explosion models with as few adjustable parameters as possible.

From the point of view of cosmology, in particular the planned use of SNe Ia as high-precision tools to map out the equation of state of the universe, some of the most urgent questions that supernova theorists and observers must answer 
are: a) What are the progenitors?, b)Is there only one class of explosions or many?, c) What is the physics that governs the diversity of SNe Ia?, and d) How robust are the correlations between peak brightness, light curve shape, and spectral features with respect to, say, multidimensional mixing? This is a challenging program that, apart from cosmology, involves a lot of fascinating physics.

Much progress has been made in recent years in the field of multidimensional explosion models. We give a very brief overview of the currently discussed explosion scenarios in Sec. (0.2) (for more details and references, see [6]) and describe our approaches to simulating Chandrasekhar mass deflagration models in two and three dimensions in Sec. (0.3).

\subsection{Explosion Scenarios}

The lack of hydrogen and presence of silicon in SN Ia spectra, the rate of the light curve decline powered by decaying nickel, and the inferred minimum age of some SN Ia progenitors are all consistent with thermonuclear explosions of $\mathrm{C}+\mathrm{O}$ white dwarf stars [7]. In order to trigger the explosion, the star is believed to accrete matter from a binary companion until critical conditions are reached. The various explosion scenarios below differ mainly in the rate and composition of the accreted material, the mass of the white dwarf when it explodes, the location of the ignition, and the propagation mode of the burning front.

The strong temperature dependence of the nuclear reaction rates, $\dot{S} \sim T^{12}$ at $T \approx 10^{10} \mathrm{~K}$, confines the nuclear burning to microscopically thin layers that propagate either conductively as subsonic deflagrations ("flames") or by shock compression as supersonic detonations. Both modes are linearly unstable to spatial perturbations. In the nonlinear regime, the burning fronts are either stabilized by forming a cellular structure or become fully turbulent - either way, the total burning rate increases as a result of flame surface growth. Neither flames nor detonations can be resolved in explosion simulations on stellar scales and therefore have to be represented by numerical models.

\subsubsection{Chandrasekhar Mass Explosion Models}

Given the overall homogeneity of SNe Ia, the good agreement of parameterized 1D models with observed spectra and light curves, and their reasonable nucleosynthetic yields, the bulk of normal SNe Ia is generally assumed to consist of exploding $\mathrm{C}+\mathrm{O}$ white dwarfs that have reached the Chandrasekhar mass, $\mathrm{M}_{\mathrm{ch}}$, by accretion of hydrogen or helium that burns stably to carbon and oxygen. Flame ignition takes place near the center following roughly $10^{3}$ years of core convection. There is no clear identification of natural progenitor systems, but supersoft X-ray sources (SSXS) look relatively promising [8].

\section{Prompt detonation}

The first hydrodynamical simulation of an exploding $\mathrm{M}_{\mathrm{ch}}$-white dwarf [9] assumed that the thermonuclear combustion commences as a detonation wave, 
consuming the entire star at the speed of sound. Given no time to expand prior to being burned, the $\mathrm{C}+\mathrm{O}$ material in this scenario is transformed almost completely into iron-peaked nuclei and thus fails to produce significant amounts of intermediate mass elements, in contradiction to observations. It is for this reason that $\mathrm{M}_{\mathrm{ch}}$-explosions are believed to begin in the deflagration (flame) mode.

\section{Pure turbulent deflagration}

Once ignited, a subsonic thermonuclear flame becomes highly convoluted as a result of turbulence produced by the Rayleigh-Taylor instability (buoyancy) and the secondary Kelvin-Helmholtz instability (shear) along the flame front. It continues to burn through the star until it either transitions into a detonation or is quenched by expansion.

By far the most simulations to date are spherically symmetric, ignoring the multidimensionality of the flame and treating the turbulent flame speed $S_{\mathrm{t}}$ as a free parameter. These studies essentially agree that good agreement with the observations is obtained if $S_{\mathrm{t}}$ accelerates up to roughly $30 \%$ of the sound speed.

In multiple dimensions, the problem of simulating turbulent deflagrations has two aspects: the representation of the thin, propagating surface separating hot and cold material with different densities, and the prescription of the local propagation velocity $S_{\mathrm{t}}(\Delta)$ of this surface as a function of the hydrodynamical state of the large-scale calculation with numerical resolution $\Delta$. Our solution to this problem is sketched in Sec. (0.3); for a different approach see [10].

Most authors agree that the turbulent flame speed decouples from microphysics on large enough scales and becomes dominated by essentially universal hydrodynamical effects, making the scenario intrinsically robust. A noteworthy exception is the location and number of ignition points that significantly influences the explosion outcome and may be a possible candidate for the mechanism giving rise to the explosion strength variability. Other possible sources of variations include the ignition density and the accretion rate of the progenitor system. All of these effects may potentially vary with composition and metallicity and can therefore account for the dependence on the progenitor stellar population.

\section{Delayed detonation}

Turbulent deflagrations can sometimes be observed to undergo spontaneous transitions to detonations (deflagration-detonation transitions, DDTs) in terrestrial combustion experiments. It was suggested that DDTs may occur in the late phase of a $\mathrm{M}_{\mathrm{ch}}$-explosion, providing an elegant explanation for the initial slow burning required to pre-expand the star, followed by a fast combustion mode that produces large amounts of high-velocity intermediate mass elements [11, 12]. Many 1D simulations have meanwhile demonstrated the capability of the delayed detonation scenario to provide good fits to SN Ia spectra and light curves, as well as reasonable nucleosynthesis products. In the best fit models,

the initial flame phase has a rather slow velocity of roughly one percent of the sound speed and transitions to detonation at a density of $\rho_{\mathrm{DDT}} \approx 10^{7} \mathrm{~g} \mathrm{~cm}^{-3}$. 
The transition density was also found to be a convenient parameter to explain the observed sequence of explosion strengths 13].

On the minus side, obtaining a DDT in unconfined media without walls or obstacles relies on local quenching of the thermonuclear burning in a region that is many orders of magnitude larger than the flame thickness [14, 15. This is difficult to achieve by turbulent strain alone [16]. Furthermore, multidimensional simulations indicate that the turbulent flame speed is closer to $30 \%$ than $1 \%$ of the speed of sound, so that delayed detonations seem no longer to be needed from the energetic point of view (although they may be beneficial for removing unburned material near the core) [10, 17.

In another variety of the delayed detonation scenario, the first turbulent deflagration phase fails to release enough energy to unbind the star which subsequently pulses and triggers a detonation upon recollapse ("pulsational delayed detonation"). All pulsational models are in conflict with current multidimensional simulations that predict an unbound star after the first deflagration phase.

\subsubsection{Sub-Chandrasekhar Mass Models}

$\mathrm{C}+\mathrm{O}$ white dwarfs below the Chandrasekhar mass do not reach the critical density and temperature for explosive carbon burning in the core and need to be ignited by an external trigger. Detonations in the accreted He layer were suggested to drive a strong enough shock into the $\mathrm{C}+\mathrm{O}$ core to initiate a secondary carbon detonation. These so-called edge-lit detonations might explain the class of very weak, subluminous explosions such as SN 1991bg. They are favored mostly by the statistics of possible SN Ia progenitor systems [18] and by the straightforward explanation of the one-parameter strength sequence in terms of the white dwarf mass. However, their ejecta structure is characterized almost inevitably by an outer layer of high-velocity $\mathrm{Ni}$ and $\mathrm{He}$ above the intermediate mass elements and the inner Fe/Ni core. Therefore, these models appear to disagree photometrically and spectroscopically with observations, but more work on the explosion physics is needed to come to a final conclusion.

\subsubsection{Merging White Dwarfs}

The merging white dwarf (or "double degenerate") scenario has to overcome the crucial problem of avoiding accretion-induced collapse before it can be seriously considered as a SN Ia candidate. If the accretion rate of $\mathrm{C}+\mathrm{O}$ onto the remaining white dwarf is larger than a few times $10^{-6} \mathrm{M}_{\odot} \mathrm{yr}^{-1}$, the most likely outcome is off-center carbon ignition leading to an inward propagating flame that converts the star into $\mathrm{O}+\mathrm{Ne}+\mathrm{Mg}$. This configuration, in turn, is gravitationally unstable owing to electron capture onto ${ }^{24} \mathrm{Mg}$ and will undergo accretion-induced collapse to form a neutron star. Dimensional analysis of the expected turbulent viscosity suggests that it is very difficult to avoid such high accretion rates [19].

Its key strengths are a plausible explanation for the progenitor history yielding reasonable predictions for SN Ia rates, the straightforward explanation of the

absence of $\mathrm{H}$ and $\mathrm{He}$ in SN Ia spectra, and the existence of a simple parameter for the explosion strength family (i.e., the mass of the merged system). 


\subsection{Multidimensional $\mathrm{M}_{\mathrm{ch}}$-Models}

In this section, we describe multidimensional simulations of what we consider the best model for the majority of SN Ia events, i.e. the pure turbulent deflagration model of Sec. (0.2.1) (see [17 for details). Our basic assumptions are as follows: the initial model is a cold white dwarf with the Chandrasekhar mass, consisting of equal amounts of carbon and oxygen. Flame ignition starts in the inner $\sim 150 \mathrm{~km}$ of the star and the initial flame geometry acts as our principal free parameter. No deflagration-detonation-transition is assumed to occur.

\subsubsection{Modeling of the turbulent combustion front}

The initial mixture consists of ${ }^{12} \mathrm{C}$ and ${ }^{16} \mathrm{O}$ at low temperatures. Because of the electron degeneracy the fuel temperature is nearly decoupled from the rest of the thermodynamic quantities, and since temperature is not used to determine the initial reaction rates, its exact value is unimportant.

When the flame passes through the fuel, carbon and oxygen are converted to ash, which has different compositions depending on the density of the unburned material. At high densities a mixture of ${ }^{56} \mathrm{Ni}$ and $\alpha$-particles in nuclear statistic equilibrium (NSE) is synthesized. At lower densities burning only produces intermediate mass elements, which are represented by ${ }^{24} \mathrm{Mg}$. Once the density drops below $10^{7} \mathrm{~g} \mathrm{~cm}^{-3}$, no burning takes place.

In the material burned to NSE, the proportion of ${ }^{56} \mathrm{Ni}$ and $\alpha$-particles changes depending on density and temperature even after the flame has processed the material.

The transition densities from NSE to incomplete burning, as well as from incomplete burning to flame extinction were derived from data of a W7 run provided by K. Nomoto. This approach is rather phenomenological, and since these densities can have a potentially large impact on the simulation outcome it will have to be re-examined in a thorough manner.

The numerical representation of the thermonuclear reaction front (i.e. the location where the "fast" reactions take place) is described in detail in [20]. The flame front is associated with the zero level set of a function $G(\vec{r}, t)$, whose temporal evolution is given by

$$
\frac{\partial G}{\partial t}=-\left(\vec{v}_{u}+s_{u} \vec{n}\right)(-\vec{n}|\vec{\nabla} G|)
$$

where $\vec{v}_{u}$ and $s_{u}$ denote the fluid and flame propagation velocity in the unburned material ahead of the front, and $\vec{n}$ is the front normal pointing towards the fuel. In our case, $s_{u}$ identified with by the effective turbulent flame speed on the scale of the grid resolution, $S_{\mathrm{t}}(\Delta)$ (see below). The advection of $G$ caused by the fluid motions is treated by the piecewise parabolic method which is also used by our code to integrate the Euler equations. After each time step, the front is additionally advanced by $s_{u} \Delta t$ normal to itself.

This equation is only applied in the close vicinity of the front, whereas in the other regions $G$ is adjusted such that

$$
|\vec{\nabla} G|=1
$$


The source terms for energy and composition due to the fast thermonuclear reactions in every grid cell are determined as follows:

$$
\begin{aligned}
X_{\text {Ashes }}^{\prime} & =\max \left(1-\alpha, X_{\text {Ashes }}\right) \\
X_{\text {Fuel }}^{\prime} & =1-X_{\text {Ashes }}^{\prime} \\
e_{\text {tot }}^{\prime} & =e_{\text {tot }}+q\left(X_{\text {Ashes }}^{\prime}-X_{\text {Ashes }}\right)
\end{aligned}
$$

where $\alpha$ is the volume fraction of the cell occupied by unburned material; this quantity can be determined from the values of $G$ in the cell and its neighbors. The quantity $q$ represents the specific energy release of the total reaction.

All multidimensional simulations of exploding white dwarfs share the problem that it is impossible to resolve all hydrodynamically unstable scales. The consequence is that the simulated thermonuclear flame can only develop structures on the resolved macroscopic scales, while the real reaction front will be folded and wrinkled on much finer scales. Simply neglecting the surface increase on sub-grid scales would lead to an underestimation of the energy generation rate, which is not acceptable; therefore a model for a turbulent flame speed $S_{\mathrm{t}}$ is required to compensate this effect.

For the case of very strong turbulence it has been shown that $S_{\mathrm{t}}$ decouples from the laminar flame speed and is proportional to the turbulent velocity fluctuations $v^{\prime}$. In our simulations, $v^{\prime}(\Delta)$ is determined by using the sub-grid model introduced to SN Ia simulations by [21]. For the presented calculations a few corrections were applied (see [17]).

\subsubsection{Two-dimensional resolution study}

To study the robustness of our code with respect to a change of the numerical resolution, simulations were performed with grid sizes of $128^{2}, 256^{2}, 512^{2}$ and $1024^{2}$ cells, whose corresponding resolutions in the uniform inner part of the grid were $2 \cdot 10^{6} \mathrm{~cm}, 10^{6} \mathrm{~cm}, 5 \cdot 10^{5} \mathrm{~cm}$ and $2.5 \cdot 10^{5} \mathrm{~cm}$. The initial flame geometry (called c3_2d) used for all these calculations is identical to the setup C3 presented by [22]: the matter within a radius of $1.5 \cdot 10^{7} \mathrm{~cm}$ from the stellar center was incinerated, and the surface of the burned region was perturbed to accelerate the development of Rayleigh-Taylor instabilities.

Fig. 1 1 shows the energy release of the models; except for the run with the lowest resolution, the curves are nearly identical in the early and intermediate explosion stages. Simulation c3_2d_128 exhibits a very slow initial energy increase and does not reach the same final level as the other models. Most likely this is due to insufficient resolution, which leads to a very coarsely discretized initial front geometry and thereby to an underestimation of the flame surface. From this result it can be deduced that all supernova simulations performed with our code should have a central resolution of $10^{6} \mathrm{~cm}$ or better.

Overall, our model for the turbulent flame speed appears to compensate the lack of small structures in the front very well. 


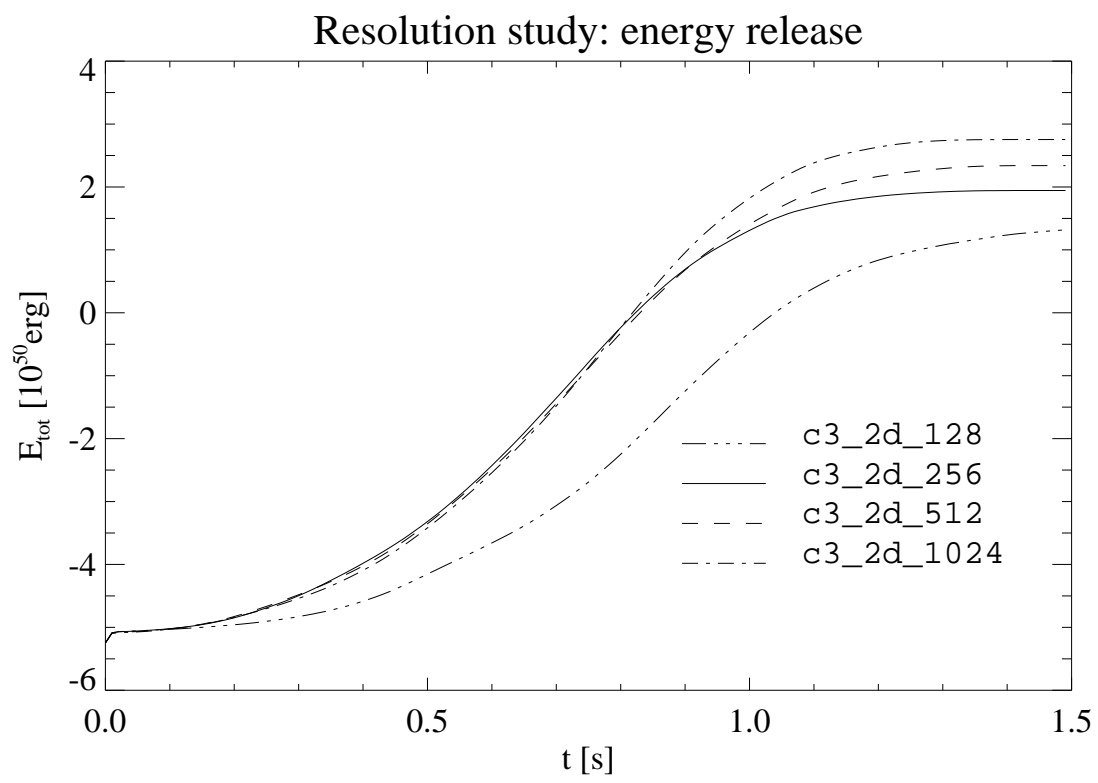

Figure 1: Time evolution of the total energy for the initial flame geometry c3_2d and different resolutions. During the early and intermediate explosion stages there is excellent agreement between the better resolved simulations.

\subsubsection{Three-dimensional simulation}

In order to compare two- and three-dimensional simulations directly, a 3D calculation was performed using the same initial conditions as given in Sect. 0.3.2. For this purpose the initial two-dimensional flame location was rotated by 90 degrees around the $z$-axis and mapped onto the three-dimensional Cartesian grid consisting of $256^{3}$ cells with a central resolution of $10^{6} \mathrm{~cm}$. Only one octant of the white dwarf was simulated and mirror symmetry was assumed with respect to the coordinate planes.

The initial configuration, as well as snapshots at later times, are shown in Fig. 2. Obviously, the initial axisymmetry is lost after $0.2-0.3 \mathrm{~s}$, although no explicit perturbation in $\varphi$-direction was applied to the front. This happens because the initial flame geometry cannot be mapped perfectly onto a Cartesian grid and therefore the front is not transported with exactly the same speed for all $\varphi$. During the next few tenths of a second, these small deviations cause the formation of fully three-dimensional Rayleigh-Taylor-mushrooms, leading to a strong convolution of the flame. As expected, this phenomenon has a noticeable influence on the explosion energetics; this is illustrated in Fig. 3. Before the loss of axial symmetry in c3_3d_256, the total energy evolution is almost identical for both simulations, which strongly suggests that the two- and three-dimensional forms of the employed turbulence and level set models are consistent, i.e. that no errors were introduced during the extension of these models to three dimensions. In the later phases the 3D model releases more energy as a direct consequence 

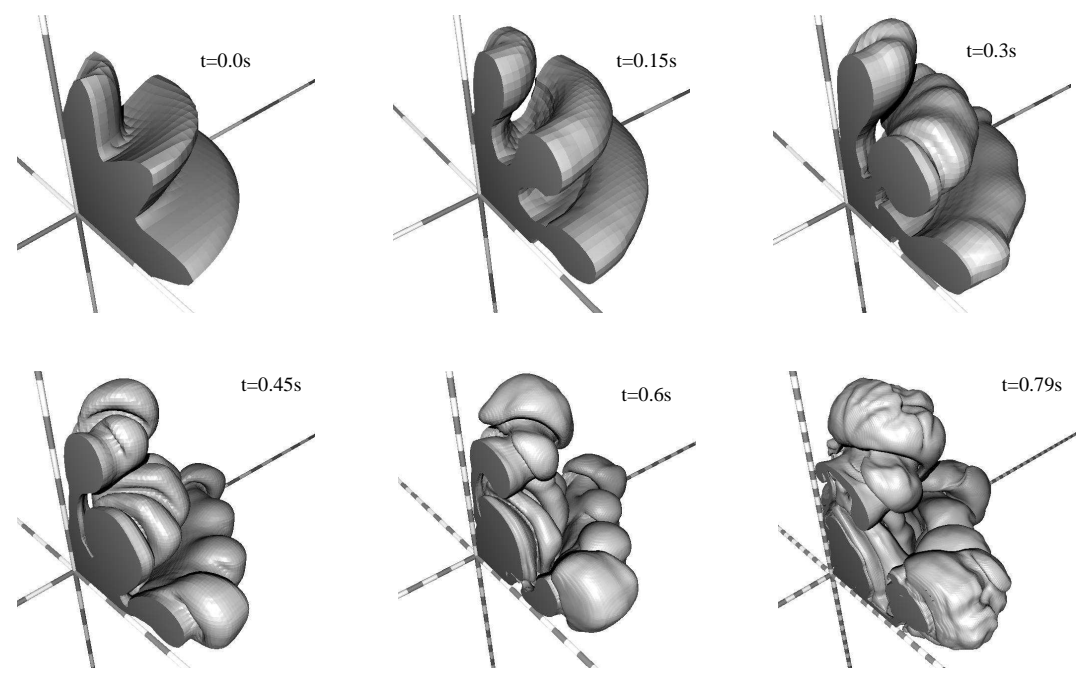

Figure 2: Snapshots of the flame front for a centrally ignited three-dimensional scenario. One ring on the coordinate axes corresponds to $10^{7} \mathrm{~cm}$.

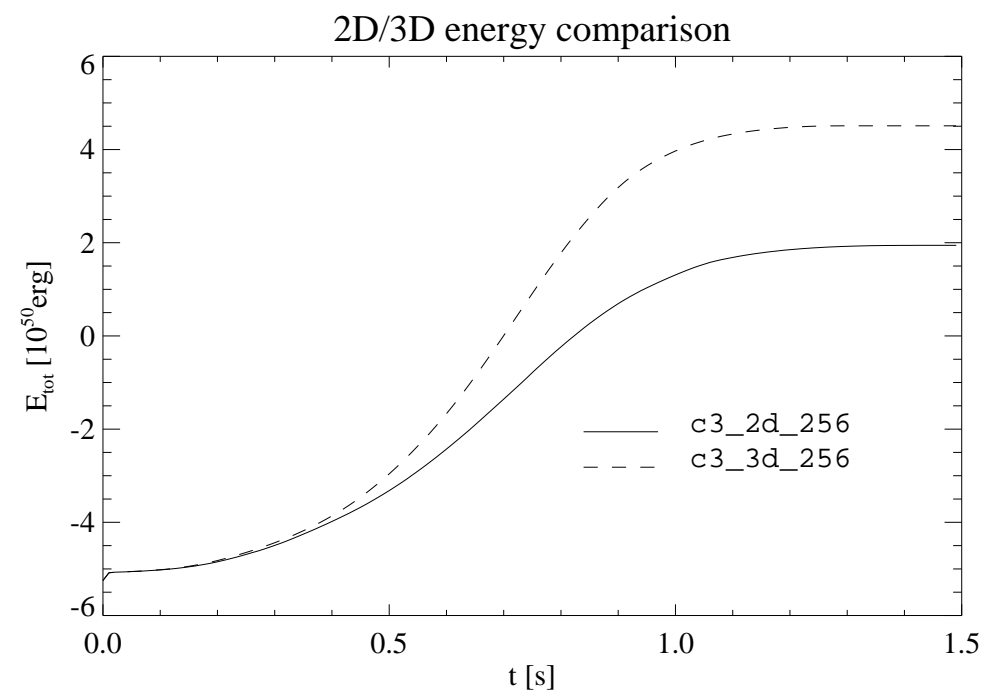

Figure 3: Comparison of the explosion energy for identical initial conditions and resolution in two and three dimensions. After the loss of axial symmetry (at $t \approx 0.3 \mathrm{~s}$ ) the larger flame surface in the three-dimensional model leads to more vigorous burning. 
of the surface increase shown in Fig. 2.

\subsubsection{Discussion and conclusions}

The white dwarf becomes unbound in all of our models, which implies that no recontraction (and hence no pulsational detontation) occurs. Nevertheless only the three-dimensional model results in a powerful enough explosion to qualify

as a typical SN Ia; the two-dimensional scenarios are too weak to accelerate the ejecta to the speeds observed in real events and produce too little nickel to power a standard SN Ia light curve.

The 3D calculation is a good candidate for typical SN Ia explosions, at least with respect to explosion strength and remnant composition. The produced nickel mass of $0.53 \mathrm{M}_{\odot}$ falls well into the range of $\approx 0.45-0.7 \mathrm{M}_{\odot}$ determined by [23] for several typical events, and it can be deduced from the amount of $0.18 \mathrm{M}_{\odot}$ of "magnesium" in the ejecta that enough intermediate mass elements were synthesized to explain the observed spectral features.

Qualitatively, our results for the explosion energetics are in relatively good agreement with recent simulations performed by [10], employing different numerical models and initial conditions. We interpret this as an inherent robustness of the Chandrasekhar mass deflagration scenario.

This development is a major step towards constructing self-consistent models for type Ia supernovae. The remaining free parameters are chosen according to our best understanding of the unresolved physics without any reference to obtaining "good" explosions. As there will always be relevant unresolved scales in this problem, we need to keep improving our understanding by performing numerical experiments of turbulent thermonuclear combustion on microscopic and intermediate scales. Nevertheless, our results make us optimistic that multidimensional models will soon allow us to understand how type Ia supernovae really work.

\section{Acknowledgements}

We thank Bruno Leibundgut and Paolo Mazzali for helpful discussions. 


\section{Bibliography}

[1] A. G. Riess, A. V. Filippenko, P. Challis, A. Clocchiatti, A. Diercks, P. M. Garnavich, R. L. Gilliland, C. J. Hogan, S. Jha, R. P. Kirshner, B. Leibundgut, M. M. Phillips, et al., Astron. J. 116, 1009 (1998).

[2] S. Perlmutter, G. Aldering, G. Goldhaber, R. A. Knop, P. Nugent, P. G. Castro, S. Deustua, S. Fabbro, A. Goobar, D. E. Groom, I. M. Hook, A. G. Kim, et al., Astrophys. J. 517, 565 (1999).

[3] J. C. Niemeyer and J. W. Truran, eds., Type Ia Supernovae: Theory and Cosmology (Cambridge Univ. Press, Cambridge, 2000).

[4] B. Leibundgut, Ann. Rev. Astron. Astrophys. 39, 67 (2001).

[5] A. V. Filippenko and A. G. Riess, in Niemeyer and Truran [3].

[6] W. Hillebrandt and J. C. Niemeyer, Ann. Rev. Astron. Astrophys. 38, 191 (2000).

[7] F. Hoyle and W. A. Fowler, Astrophys. J. 132, 565 (1960).

[8] M. Livio, in Niemeyer and Truran [3].

[9] W. D. Arnett, Astrophys. Space Sci. 5, 180 (1969).

[10] A. Khokhlov (2001), astro-ph/0008463.

[11] A. M. Khokhlov, Astron. Astrophys. 245, 114 (1991).

[12] S. E. Woosley and T. A. Weaver, in Supernovae, Les Houches Session LIV, edited by J. Audouze, S. Bludman, R. Mochovitch, and J. Zinn-Justin (Elsevier, Amsterdam, 1994), p. 63.

[13] P. Hoeflich and A. Khokhlov, Astrophys. J. 457, 500 (1996).

[14] J. C. Niemeyer and S. E. Woosley, Astrophys. J. 475, 740 (1997).

[15] A. M. Khokhlov, E. S. Oran, and J. C. Wheeler, Astrophys. J. 478, 678 (1997).

[16] J. C. Niemeyer, Astrophys. J. Lett. 523, L57 (1999). 
[17] M. Reinecke, W. Hillebrandt, and J. C. Niemeyer, Astron. Astrophys. (accepted) (2002).

[18] L. Yungelson and M. Livio, Astrophys. J. 497, 168 (1998).

[19] R. Mochkovitch and M. Livio, Astron. Astrophys. 236, 378 (1990).

[20] M. Reinecke, W. Hillebrandt, J. C. Niemeyer, R. Klein, and A. Gröbl, Astron. Astrophys. 347, 724 (1999).

[21] J. C. Niemeyer and W. Hillebrandt, Astrophys. J. 452, 769 (1995).

[22] M. Reinecke, W. Hillebrandt, and J. C. Niemeyer, Astron. Astrophys. 347, 739 (1999).

[23] G. Contardo, B. Leibundgut, and W. D. Vacca, Astron. Astrophys. 359, 876 (2000). 